\title{
Estrogen regulates Hippo signaling via GPER in breast cancer
}

\author{
Xin Zhou, ${ }^{1,2,3}$ Shuyang Wang, ${ }^{4}$ Zhen Wang, ${ }^{1,2,3}$ Xu Feng, ${ }^{1,2,3}$ Peng Liu, ${ }^{1,2,3}$ Xian-Bo Lv, ${ }^{1,2,3}$ Fulong Li, ${ }^{1,2,3}$ Fa-Xing Yu, ${ }^{5}$ Yiping Sun, ${ }^{1,2}$ \\ Haixin Yuan, ${ }^{1,2}$ Hongguang Zhu, ${ }^{4}$ Yue Xiong, ${ }^{2,3,6}$ Qun-Ying Lei, ${ }^{1,2}$ and Kun-Liang Guan ${ }^{1,2,7}$ \\ ${ }^{1}$ Key Laboratory of Metabolism and Molecular Medicine, Ministry of Education, and Department of Biochemistry and Molecular Biology, Fudan University Shanghai Medical College, Collaborative Innovation Center \\ of Cenetics and Development, Shanghai, China. ${ }^{2}$ Molecular and Cell Biology Lab, Institutes of Biomedical Sciences, and ${ }^{3}$ School of Life Science, Fudan University, Shanghai, China. ${ }^{4}$ Department of Pathology, \\ Fudan University Shanghai Medical College, Shanghai, China. ${ }^{5}$ Children's Hospital and Institutes of Biomedical Sciences, Fudan University, Shanghai, China. ${ }^{6}$ Department of Biochemistry and Biophysics, \\ Lineberger Comprehensive Cancer Center, University of North Carolina at Chapel Hill, Chapel Hill, North Carolina, USA. 'Department of Pharmacology and Moores Cancer Center, UCSD, La Jolla, California, USA.
}

\begin{abstract}
The $\mathrm{G}$ protein-coupled estrogen receptor (GPER) mediates both the genomic and nongenomic effects of estrogen and has been implicated in breast cancer development. Here, we compared GPER expression in cancerous tissue and adjacent normal tissue in patients with invasive ductal carcinoma (IDC) of the breast and determined that GPER is highly upregulated in cancerous cells. Additionally, our studies revealed that GPER stimulation activates yes-associated protein 1 (YAP) and transcriptional coactivator with a PDZ-binding domain (TAZ), 2 homologous transcription coactivators and key effectors of the Hippo tumor suppressor pathway, via the G $\alpha q-11$, PLC $\beta / P K C$, and Rho/ROCK signaling pathways. TAZ was required for GPER-induced gene transcription, breast cancer cell proliferation and migration, and tumor growth. Moreover, TAZ expression positively correlated with GPER expression in human IDC specimens. Together, our results suggest that the Hippo/YAP/TAZ pathway is a key downstream signaling branch of GPER and plays a critical role in breast tumorigenesis.
\end{abstract}

\section{Introduction}

Estrogen stimulates mammary epithelial cell proliferation by mainly activating the nuclear estrogen receptors $\mathrm{ER} \alpha$ and $\mathrm{ER} \beta$, which are sequence-specific DNA-binding transcription factors, to regulate gene expression $(1,2)$. Estrogen has paramount importance in breast cancer. As a result, hormone therapy (inhibition of estrogen signaling or estrogen production) is a common clinical treatment for estrogen receptor-positive (ER-positive) breast cancer. Besides ERs, the seven-transmembrane $G$ protein-coupled estrogen receptor (GPER, also known as GPR30), which is a G protein-coupled receptor (GPCR), can also be activated by estrogen to induce rapid effects such as intracellular calcium mobilization $(3,4)$.

GPCR, the largest cell surface receptor family encoded by the human genome, is involved in a wide range of physiological regulatory activities and represents the target of $50 \%$ of all prescription drugs (5). Notably, recent cancer genomic studies have shown that the GPCR has an important role in cancer development, with nearly $20 \%$ of human cancers harboring mutations in GPCRs (6). Stimulation of GPCRs by upstream ligands can initiate numerous downstream signaling pathways, including PLC/PKC, Ras/MAP kinase, PI3K/AKT, and mTOR, to promote cell proliferation (5).

Authorship note: Xin Zhou and Shuyang Wang contributed equally to this work. Note regarding evaluation of this manuscript: Manuscripts authored by scientists associated with Duke University, The University of North Carolina at Chapel Hill, Duke-NUS, and the Sanford-Burnham Medical Research Institute are handled not by members of the editorial board but rather by the science editors, who consult with selected external editors and reviewers.

Conflict of interest: The authors have declared that no conflict of interest exists. Submitted: October 17, 2014; Accepted: March 12, 2015.

Reference information: / Clin Invest. 2015;125(5):2123-2135. doi:10.1172/JCI79573.
However, compared with the well-established GPCR signaling cascades, much less is known about the molecular bases of GPER in breast cancer, although some studies have identified that GPER could transactivate the EGFR/ERK pathway through metalloproteinases and the cleaved heparin-binding $\operatorname{EGF}(7,8)$. Increasing evidence has demonstrated that estrogen promotes the proliferation, migration, and invasion of cancer cells, particularly breast cancer cells, at least in part through GPER (9-11). Some studies suggested that the expression levels of GPER positively correlate with poor outcome in breast cancer $(12,13)$; however, others indicated that GPER is downregulated in breast cancer (13-15). The precise function and mechanism of GPER in breast cancer remains to be determined.

The Hippo tumor suppressor pathway is evolutionarily conserved from Drosophila to mammals and serves as a key regulator of tissue growth and organ size by limiting cell proliferation and migration and promoting apoptosis $(16,17)$. Dysregulation of the Hippo pathway is associated with human cancers (18). The central components of the Hippo pathway contain a kinase cascade (consisting of MST1/2 and LATS1/2) and the downstream transcription coactivators YAP/ TAZ (17). MST1/2 phosphorylate and activate LATS1/2, which then phosphorylate and inhibit YAP/TAZ (19-21). The phosphorylated YAP/TAZ are sequestrated in the cytoplasm by binding to 14-3-3 or degraded via the ubiquitin-proteasome system upon additional phosphorylation (19, 20, 22-25); the dephosphorylated YAP/TAZ are localized in the nucleus, where they bind and activate the TEAD family transcription factors $(26,27)$, leading to expression of the target genes for cell proliferation, migration, and survival. Mutation, amplification, or epigenetic silencing of the Hippo pathway genes have been observed in various human cancers (18). For instance, 
A
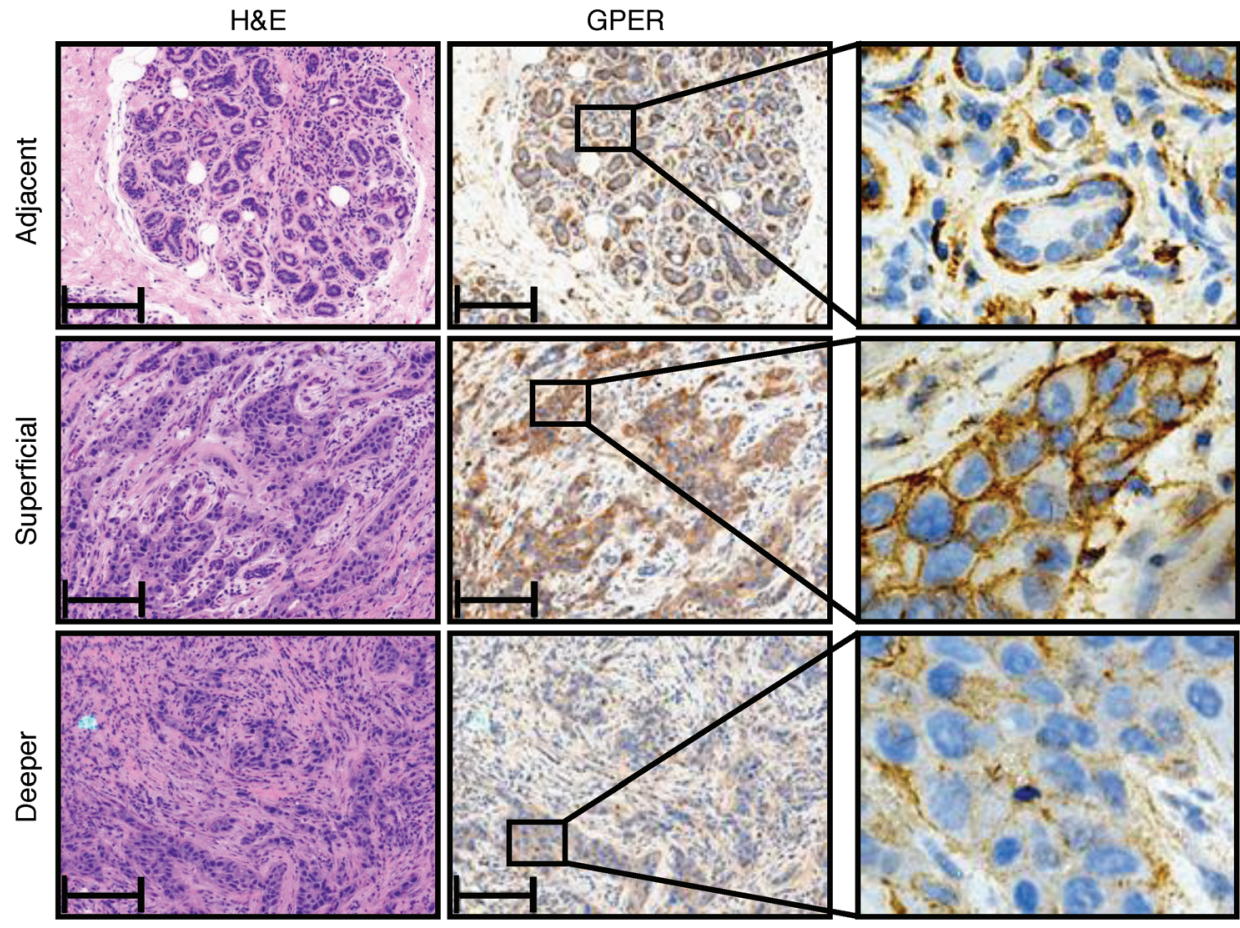

B

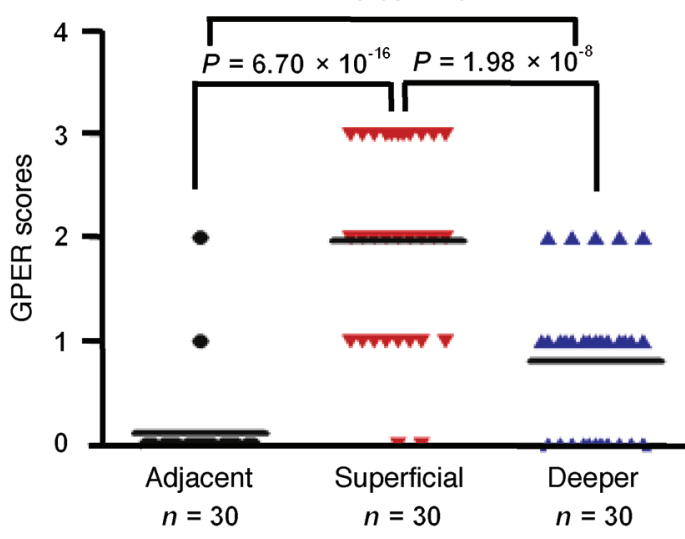

C

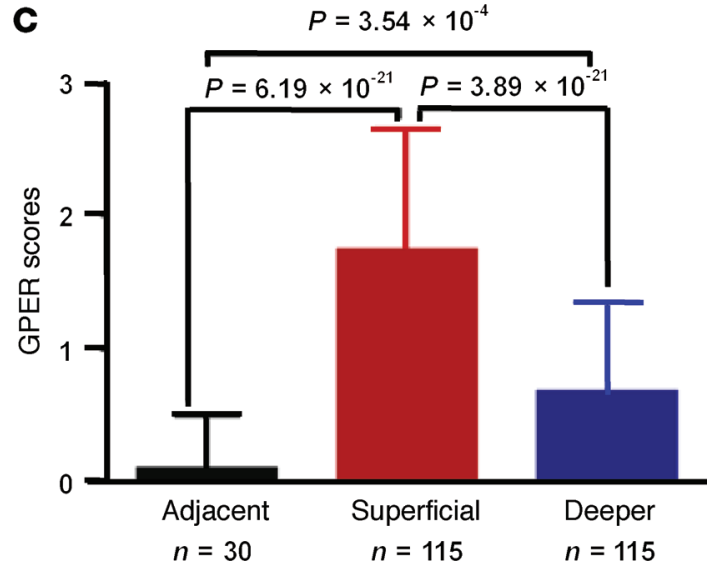

Figure 1. GPER expression is elevated in IDC of the breast. (A) IHC detection of GPER expression in a representative breast IDC sample (including both the superficial and deeper areas) and a matched adjacent normal breast tissue. Tissue slices were H\&E stained (left). The corresponding contiguous slices were stained for GPER (brown) and DNA (blue). Panels on the right show higher magnification (original magnification, $\times 400$ ) of the boxed areas in the middle panels. Scale bars: $50 \mu \mathrm{m}$. (B and C) GPER expression was elevated in IDC. GPER staining intensity was quantified using the inForm System as described in Methods. Scores of GPER expression ( 0 = lowest, 3 = highest) from 30-matched pairs of carcinoma tissues and adjacent normal breast epithelium were analyzed by 1-way ANOVA with least-significant-difference (LSD) correction. Black lines within the data points represent the mean value (B). A total of 115 breast cancer tissues were compared with 30 adjacent normal breast epithelial tissues using 1-way ANOVA with LSD correction and are shown as column plots (C). Data are represented as the mean \pm SD.

LATS2 is frequently mutated in malignant mesotheliomas (28); TAZ is overexpressed in $20 \%$ of breast cancers, especially in invasive ductal carcinoma (IDC) (29), and TAZ expression levels and activity are frequently upregulated in high-grade metastatic breast cancer (30). Interestingly, TAZ has also been implicated in the self-renewal and tumor initiation capabilities of breast cancer stem cells (30).

Recent studies by other groups and us have revealed that the Hippo/YAP/TAZ pathway is regulated by some hormones and their corresponding GPCRs $(31,32)$. Pandey and colleagues showed that GPER mediates the expression of a large number of genes in breast cancer cells (9). Interestingly, among the GPER-dependent genes described are CTGF, CYR61, EDN1, and EGR1, which are well-established YAP/TAZ target genes (26). These observations raise the possibility that GPER may regulate the Hippo/YAP/TAZ pathway to control breast cancer cell proliferation. In this study, we investigated GPER expression in IDC specimens and its role in $\mathrm{YAP} / \mathrm{TAZ}$ activation as well as in breast cancer development.

\section{Results}

GPER expression is elevated in IDC of the breast. IDC is the most common form of breast cancer, comprising $70 \%$ to $80 \%$ of all breast cancers. To investigate the potential role of GPER in breast cancer, 
we collected paired IDC specimens from 30 patients with matched adjacent normal breast tissues. IHC was carried out using a GPER antibody, the specificity of which was confirmed by antigen competition experiments (Supplemental Figure 1A; supplemental material available online with this article; doi:10.1172/JCI79573DS1). Although strong GPER staining was found in the myoepithelial cells of the adjacent normal tissues, no detectable staining (28 of 30) was found in either ductal or lobular epithelial cells (Figure 1A). In contrast, GPER-positive staining was found in 28 of 30 (93.3\%) of the cancer specimens tested (Figure 1, A and B). Interestingly, we further noticed that GPER was strongly expressed in the superficial areas in 24 of 30 (80\%) specimens that had weaker plasma membrane staining in the deeper areas of IDC samples (Figure 1, A and B). GPER expression was observed predominantly on the plasma membrane, and in the cytoplasm in some cases, no nuclear staining was observed (Figure 1A). This expression pattern is consistent with the fact that GPER serves as a membrane receptor.

IDC generally develops in ductal epithelial cells but not in myoepithelial cells. Therefore, comparison of GPER expression in ductal epithelial cells of the adjacent normal breast tissues with that in the cancer cells of the IDC specimens would be insightful. For quantification, the optical density of GPER staining was calculated using the CSI-Nuance Multispectral Tissue Imaging System and inForm Advanced Image Analysis Software (see Methods). Statistical analysis of 30 paired specimens confirmed that GPER was significantly elevated in both the superficial areas $\left(P=6.70 \times 10^{-16}\right)$ and the deeper areas $\left(P=3.65 \times 10^{-4}\right)$ of the IDC samples when compared with the normal breast ductal epithelial cells (Figure 1B). Quantitative analysis also revealed a significant difference $\left(P=1.98 \times 10^{-8}\right)$ in GPER expression levels between the superficial areas and the deeper areas of the IDC specimens (Figure 1B). To further confirm this observation, we analyzed another cohort of 96 subjects that did not have the corresponding matched adjacent normal tissues. Among the 96 specimens, 85 were large enough to contain the superficial and deeper areas of IDC samples in the same sections. We therefore combined the 85 specimens with the former 30 paired specimens for statistical analysis (Figure 1C). The results further strengthened our conclusion that GPER expression levels are significantly elevated in IDC specimens compared with those in ductal epithelial cells of normal breast tissues and that GPER expression levels are higher in the superficial areas than in the deeper areas of tumors.

The expression of GPER was then compared with prognostic parameters including tumor size, nodal status, histological grade, tumor-node-metastasis (TNM) stage, and the expression levels of $\mathrm{ER}$, progesterone receptor (PR), and human epidermal growth factor receptor 2 (HER2) (Supplemental Table 1). A significant inverse correlation was found between GPER and ER expression levels (Supplemental Figure 1, B and C). Additionally, the expression of GPER was higher in the lymph node-positive breast cancer specimens, although without reaching a level of significance $(P=0.057)$ (Supplemental Table 1). There was no correlation between the expression of GPER and other tumor characteristics (Supplemental Table 1).

Breast cancers can be divided into 5 stages -0 to IV - according to the size of the tumor $(\mathrm{T})$, the lymph node status $(\mathrm{N})$, and the metastasized phenotype (M). To determine whether GPER expression levels correlate with the progression of IDC, we analyzed the intensity of GPER in a panel of 122 IDC specimens on the basis of their stage. GPER protein levels were significantly elevated in the cancer cells of IDC specimens from all stages when compared with the ductal epithelial cells of the adjacent normal breast tissues (Supplemental Figure 1D). However, no significant alterations were observed among the different stages of IDC (Supplemental Figure 1E). Taken together, these data show that elevated GPER expression occurs at the early stage of breast tumor development, suggesting a possible role of GPER in breast IDC initiation.

Estrogen and other GPER agonists activate YAP/TAZ. It has been reported that estrogen could regulate the expression of numerous genes, including several well-characterized YAP/TAZ target genes (CTGF , CYR61, EDN1, and EGR1), through GPER (9). These observations led us to hypothesize that estrogen may activate YAP/TAZ. To this end, we tested whether E2 (17 $\beta$-estradiol), a hormone that binds to both $\mathrm{ER} \alpha / \beta$ and GPER, could activate YAP/ TAZ , 2 transcription coactivators downstream of the Hippo tumor suppressor pathway. To exclude the effect of ERs, the ER-negative SK-BR-3 breast cancer cells were examined. We found that YAP was highly phosphorylated and inactive under serum-starvation conditions. Addition of E2 induced a rapid and significant YAP dephosphorylation as determined by a phosphorylated YAP (Ser127) antibody (Figure 2A, left panel). Given that YAP activity is inhibited by phosphorylation, this result shows that E2 activates YAP in SK-BR-3 cells. OHT (4-hydroxytamoxifen) is the active metabolite of tamoxifen, which is broadly used as an antagonist of ER in hormone therapy for breast cancer $(33,34)$. However, $\mathrm{OHT}$ also functions as an agonist for $\operatorname{GPER}(7,9,35)$. Interestingly, we observed that OHT also induced YAP dephosphorylation in SK-BR-3 cells (Figure 2A, middle panel), indicating that activation of GPER, but not ER, leads to YAP dephosphorylation. Next, we examined the effect of G1, which is a selective GPER agonist without activity toward ER (36). G1 stimulation also resulted in YAP dephosphorylation (Figure 2A, right panel). Collectively, these data indicate that stimulation of GPER by E2, OHT, and G1 induces YAP dephosphorylation and activation.

TAZ is a YAP homolog that is also tightly regulated by the Hippo pathway, but it appears to play a more prominent role in breast cancer $(21,29,30)$. Moreover, the stability of TAZ is more sensitively regulated than YAP by the Hippo pathway because of its 2 phosphodegrons $(24,25)$. Notably, TAZ expression is quite low and does not respond significantly to E2, OHT, or G1 treatment in SK-BR-3 cells. Therefore, we determined TAZ expression and phosphorylation levels in another breast cancer cell line, ZR-7530. We found that $\mathrm{G} 1$ treatment led to a robust accumulation of TAZ proteins in ZR-75-30 cells (Figure 2B). G1 also induced strong TAZ dephosphorylation as determined by the phos-tag gel (Figure $2 \mathrm{~B}$ ), in which the phosphorylated TAZ ran much slower than did the unphosphorylated TAZ. Additionally, we treated Bcap-37, BT-474, ZR-75-1, and MDA-MB-361 breast cancer cells and HeLa cervical cancer cells (the expression of $\mathrm{ER} \alpha, \mathrm{ER} \beta$, and GPER in these cell lines is shown in Supplemental Figure 2A) with G1 and observed a decrease in the phosphorylation of YAP and an accumulation of TAZ protein (Supplemental Figure 2A), suggesting cell line-independent roles of GPER in YAP/TAZ activation.

To further dissect which ER is involved in YAP/TAZ activation, we silenced $E R \alpha / \beta$ and GPER in ZR-75-30 cells. As shown in Supplemental Figure 2B, G1-induced TAZ dephosphorylation and pro- 
A Effect on ER

Activation

Inhibition

Effect on GPER

Activation

Activation

Activation

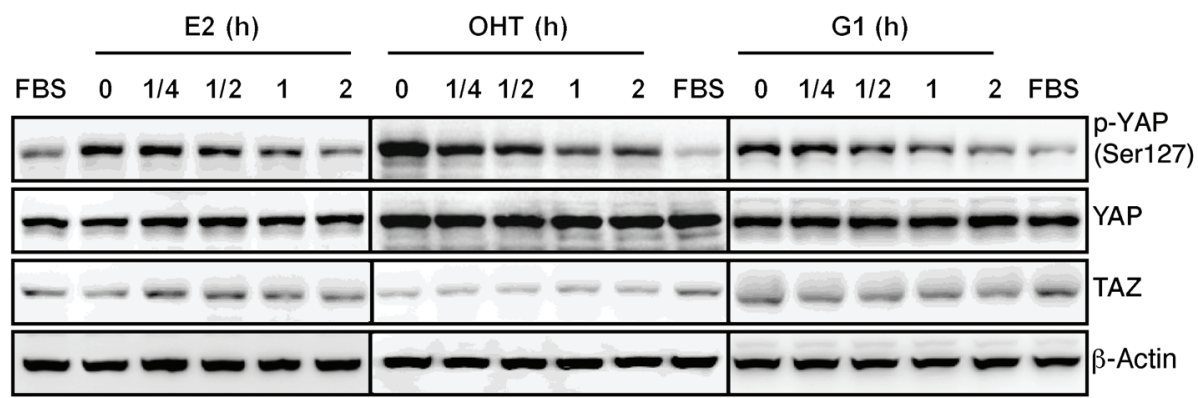

B

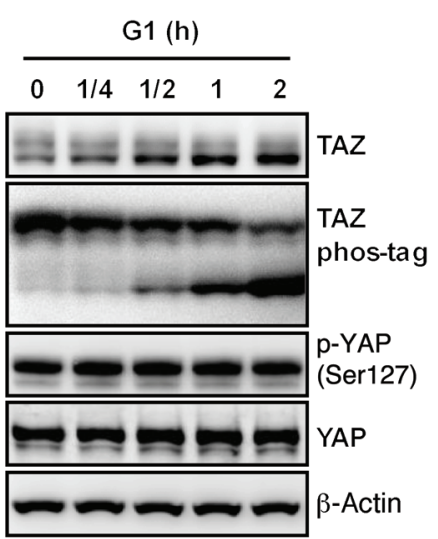

E

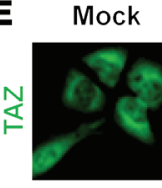

G1
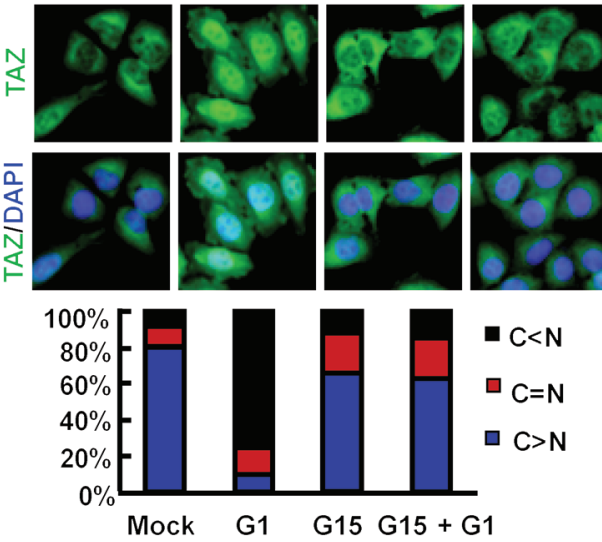

H

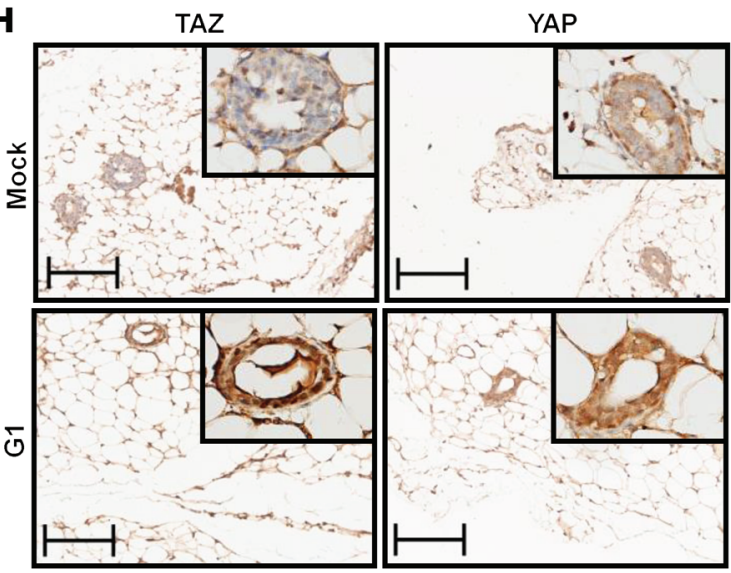

C shCtr

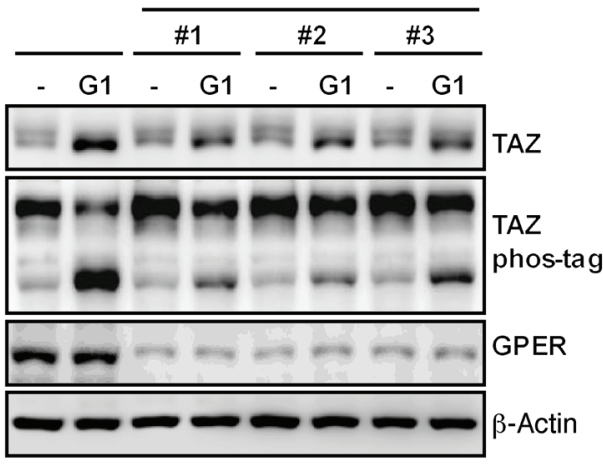

D

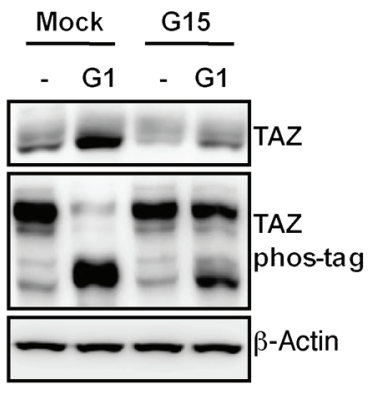

G
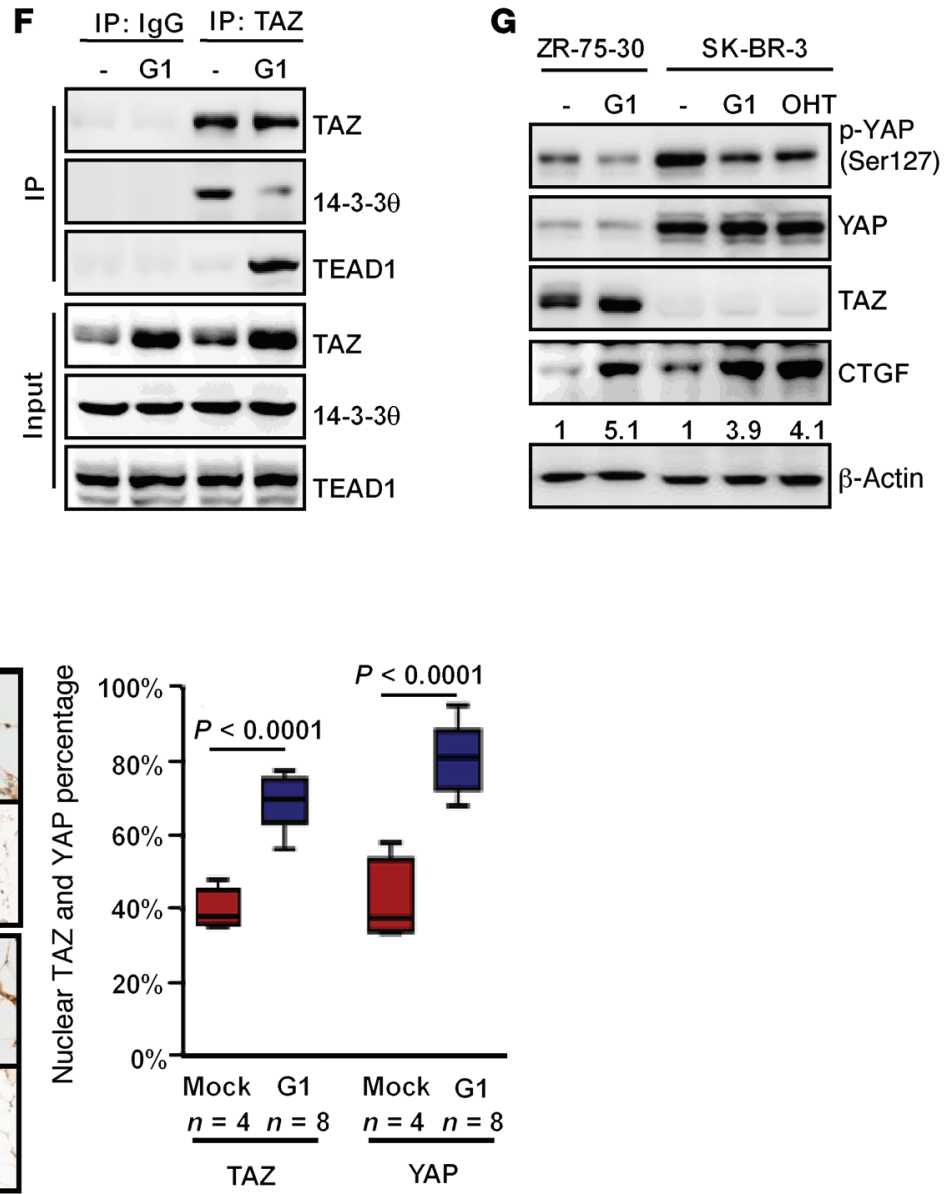
Figure 2. Stimulation of GPER activates YAP/TAZ. (A) Activation of GPER induced YAP dephosphorylation. Serum-starved SK-BR-3 cells were stimulated with $100 \mathrm{nM} \beta$-estradiol (E2), $200 \mathrm{nM} \mathrm{OHT,} \mathrm{or} 100 \mathrm{nM} \mathrm{G1.} \mathrm{Immuno-}$ blotting was performed. (B) GPER activation led to TAZ dephosphorylation and accumulation. ZR-75-30 cells were serum starved and stimulated with $100 \mathrm{nM}$ C1. TAZ phosphorylation was assessed by phos-tag gels. TAZ quantification is summarized in Supplemental Table 2. (C and D) GPER mediated TAZ activation. GPER was inhibited either by shRNAs (C) or 500 nM G15 (D), and ZR-75-30 cells were treated and immunoblotted as indicated. (E) G1 stimulated TAZ nuclear localization via GPER. Serum-starved ZR-75-30 cells were treated with G1 and/or G15, and immunofluorescence staining for TAZ was performed. Quantifications of TAZ subcellular localization from 100 randomly selected cells are shown. C, cytoplasmic; $\mathrm{N}$, nuclear. (F) $\mathrm{G} 1$ enhanced TAZ interaction with TEAD1 but inhibited TAZ interaction with 14-3-30. Serum-starved ZR-75-30 cells were treated with $\mathrm{G} 1$ for 2 hours and then subjected to immunoprecipitation with TAZ antibody. The coimmunoprecipitated TEAD1 and 14-3-30 were detected. (C) Activation of GPER increased the expression of the YAP/TAZ target gene CTCF. Serum-starved SK-BR-3 or ZR-75-30 cells were treated with $\mathrm{G} 1$ or $\mathrm{OHT}$ as indicated. The quantification of CTCF expression is shown. (H) G1 stimulated TAZ and YAP nuclear localization in mammary glands. Mice were injected with $\mathrm{G} 1$ as described in the Supplemental Methods and stained for TAZ and YAP. Scale bars: $50 \mu \mathrm{m}$. Original magnification, $\times 400$ (insets). The percentage of nuclear TAZ and YAP was analyzed by Student's $t$ test. Horizontal lines represent the median; the top and bottom of the boxes represent the 75th and 25th percentiles. Data are representative of at least 3 independent experiments. p-YAP, phosphorylated YAP.

tein accumulation were not affected in the $E R \alpha / \beta$-knockdown cells. In contrast, knockdown of GPER using 3 different shRNAs significantly suppressed the effects of G1 on TAZ dephosphorylation and protein accumulation (Figure $2 \mathrm{C}$ ). In addition, we examined the effect of G15, a G1 analog that preferentially inhibits GPER (37). We found that G15 blocked G1-induced TAZ accumulation and dephosphorylation in ZR-75-30 cells (Figure 2D). Collectively, our data show that GPER activation is responsible for YAP/TAZ dephosphorylation and activation in response to estrogen, OHT, or G1.

Subcellular localization of YAP/TAZ is tightly controlled by the Hippo pathway, as the phosphorylation of YAP on Ser127 and TAZ on Ser89 by LATS promotes 14-3-3 binding and cytoplasmic localization (19-22). The dephosphorylated YAP and TAZ translocate into the nucleus and induce gene expression by interacting with the TEA domain-containing transcription factors TEAD1-4 (26, 27). We found that $G 1$ caused significant nuclear accumulation of TAZ in ZR-75-30 cells (Figure 2E), and this effect was largely blocked by G15 (Figure 2E). As with TAZ, YAP nuclear localization was stimulated by E2, OHT, or G1 in SK-BR-3 cells (Supplemental Figure 2C). Consistent with its ability to promote TAZ dephosphorylation, G1 inhibited the interaction between TAZ and 14-3-30 and promoted the interaction between TAZ and TEAD1 in ZR-7530 cells (Figure $2 \mathrm{~F}$ ). On the basis of the above data, we conclude that stimulation of GPER activates YAP/TAZ by inducing their dephosphorylation, nuclear localization, and interaction with the target TEAD transcription factors.

To confirm the functional activation of YAP/TAZ upon GPER stimulation, we examined the expression of YAP/TAZ target genes. We found that the mRNA levels of CTGF, CYR61, EDN1, and ANKRD1 were significantly induced by G1 treatment (Supplemental Figure 2D). As expected, induction of CTGF protein expression was confirmed in 2 breast cancer cell lines upon G1 or
OHT stimulation (Figure $2 \mathrm{G}$ ). We also noticed that $\mathrm{G} 1$ increased TAZ protein levels in ZR-75-30 cells. On the other hand, SK-BR-3 cells expressed little TAZ; therefore, only YAP phosphorylation was significantly decreased by G1 stimulation, while in Bcap-37, BT-474, ZR-75-1, MDA-MB-361, and HeLa cells, both YAP and TAZ were activated in response to G1 stimulation (Supplemental Figure 2A). These results indicate that GPER stimulation usually activates YAP or TAZ; however, exactly whether YAP and/or TAZ are activated may be cell-type dependent.

To test whether GPER is a physiological regulator of YAP/ TAZ in vivo, we injected female mice with G1. As shown in Figure $2 \mathrm{H}, \mathrm{G} 1$ treatment significantly increased YAP/TAZ nuclear localization in the epithelial cells of mammary glands. In addition, we observed that G1 stimulated TAZ accumulation in utero (Supplemental Figure 2E). These data suggest that stimulation of GPER can activate $\mathrm{YAP} / \mathrm{TAZ}$ in vivo.

GPER acts through G $\alpha q / 11, P L C \beta / P K C$, and Rho/ROCK to activate TAZ. Rapid mobilization of intracellular calcium, which is widely observed upon GPER stimulation $(4,36)$, is a representative phenomenon of activation of phospholipase $C \beta$ (PLC $\beta$ ) signaling, which is directly controlled by the specific $\mathrm{G}$ protein $\mathrm{G} \alpha \mathrm{q} / 11(5,38)$. Recent studies have indicated a role of Gaq/11 in YAP/TAZ activation (31). Together, these observations suggest that $\mathrm{G} \alpha \mathrm{q} / 11$ and PLC $\beta /$ PKC pathways may play a role in GPER-initiated signaling in YAP/TAZ activation. To test this model, G $\alpha q / 11$ was knocked down by RNA interference in ZR-75-30 cells. As shown in Figure $3 \mathrm{~A}$, knockdown of $\mathrm{G} \alpha q / 11$ with 2 different siRNAs significantly blocked G1-induced dephosphorylation and protein accumulation of TAZ. Furthermore, G1-induced TAZ nuclear localization was blunted by the siRNA targeting $G \alpha q / 11$ but not by the control siRNA (Supplemental Figure 3A). Finally, G1-stimulated expression of CTGF was also effectively inhibited in G $\alpha q / 11$-knockdown cells, consistent with a functional inhibition of TAZ activity (Figure 3A). A major downstream effector of Gaq/11 is the PLC/PKC pathway $(5,38)$. A previous study also implied that PKC is one of the potential downstream effectors of GPER in rat kidney (39). As shown in Figure 3B and Supplemental Figure 3B, pretreatment with U73122 or ET-18-OCH3, two specific inhibitors of PLC $\beta$, strongly impeded G1-induced TAZ accumulation, dephosphorylation, and nuclear localization in ZR-75-30 cells. Similar effects were also observed when we treated the cells with PKC inhibitors (enzastaurin and chelerythrin chloride) (Figure $3 \mathrm{C}$ and Supplemental Figure $3 C)$. These findings led us to conclude that Gaq/11 and PLC $\beta-P K C$ function downstream of GPER to activate TAZ. In other words, $\mathrm{G} \alpha \mathrm{q} / 11$ and PLC $\beta / \mathrm{PKC}$ serve as upstream regulators of the Hippo pathway in relaying signals from GPER stimulation.

We noticed that G1 stimulation promoted stress fiber formation (Supplemental Figure 3D), which is a characteristic feature of Rho/ROCK activation. GPCR can activate Rho via Ga12/13 or Gaq/11 $(5,38)$. Previous studies have shown that Rho can activate YAP/TAZ $(40,41)$, we therefore tested the function of Rho in G1-induced TAZ activation. Pretreatment with botulinum toxin C3, a specific inhibitor of Rho GTPases, strongly blocked G1-induced TAZ accumulation in ZR-75-30 cells (Figure 3D). TAZ dephosphorylation, nuclear localization, and elevated expression of CTGF were also suppressed by C3 treatment (Figure 3D and Supplemental Figure 3D). To further support this conclusion, we 
A

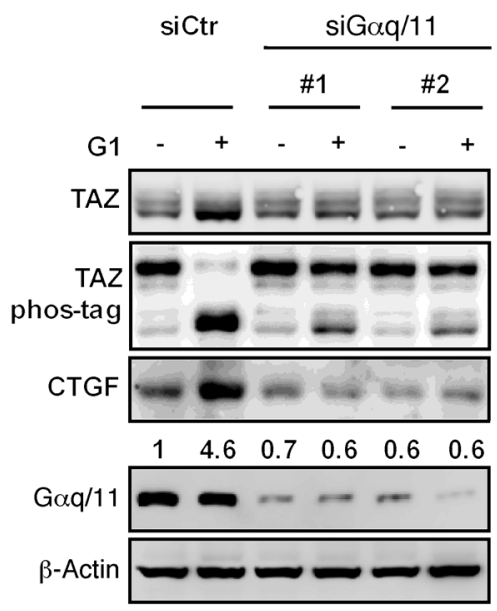

C

Enzastaurin $\quad-\quad 100 \mathrm{nM}$

Chelerythrin chloride
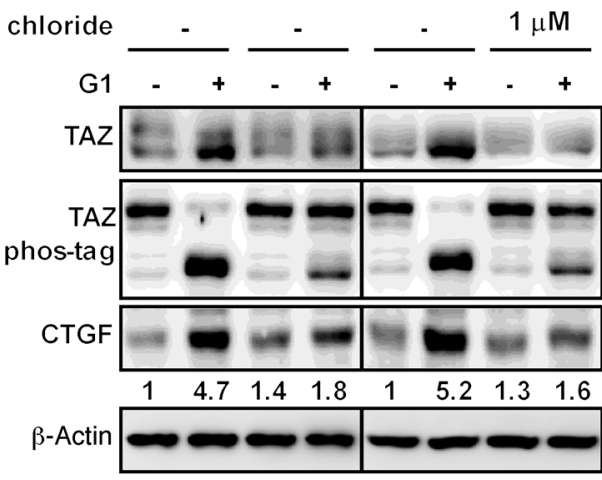

E

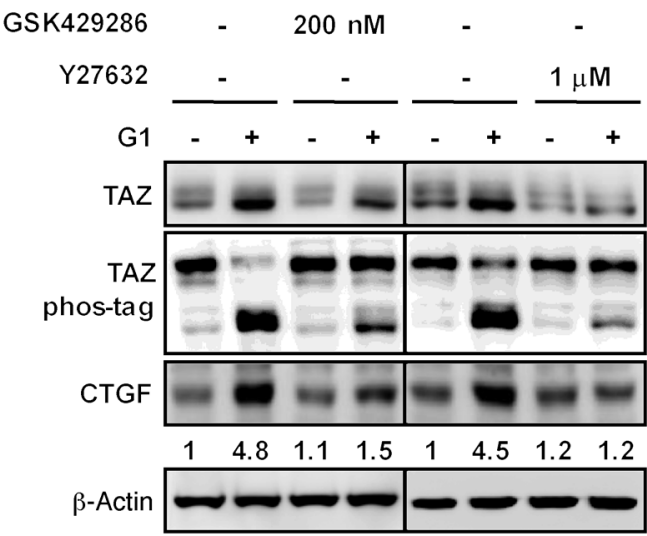

B

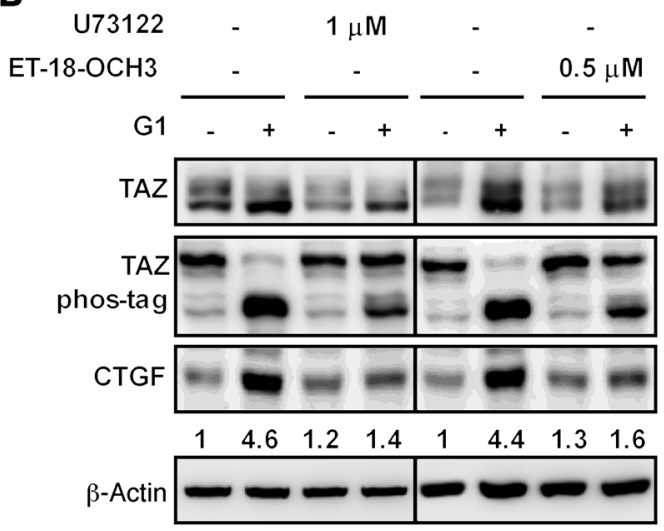

D

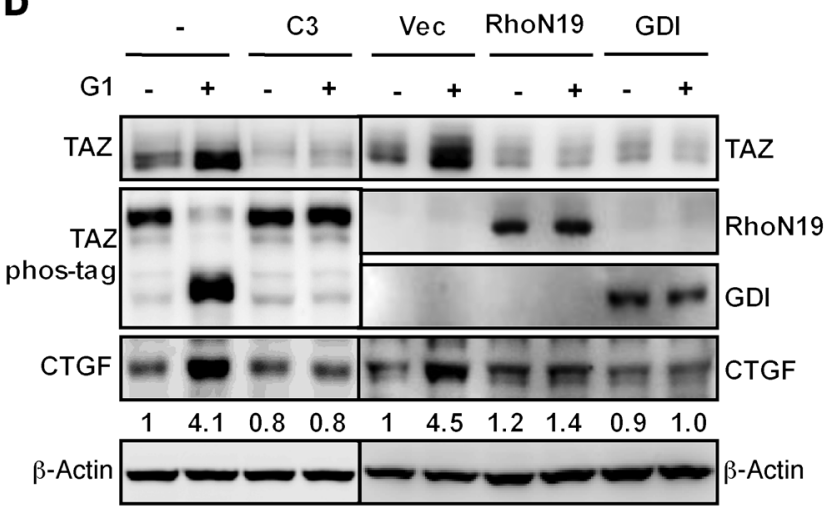

$\mathbf{F}$

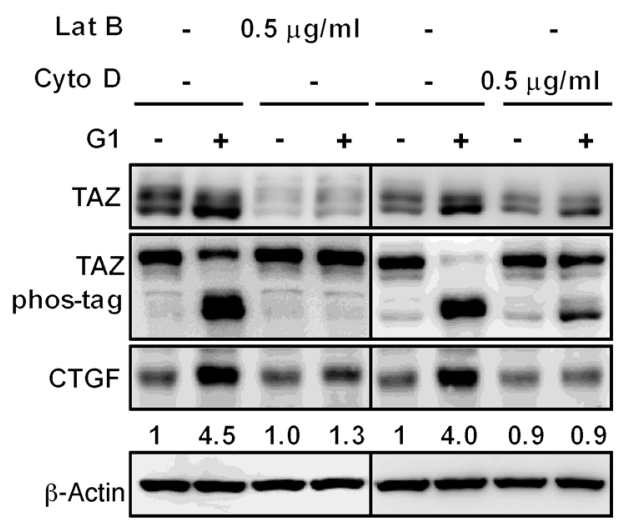

Figure 3. GPER acts through G $\alpha \mathbf{q} / 11$, PLC $\beta$-PKC, and Rho/ROCK to stimulate TAZ. (A) G $\alpha$ q/11 was required for G1 to activate TAZ. ZR-75-30 cells were transfected with control or 2 different G $\alpha q / 11$ siRNAs. After 8 hours of serum starvation, cells were treated with 100 nM G1 for 2 hours. The knockdown efficiency of $G \alpha q / 11$, TAZ protein levels and phosphorylation, and CTCF expression were determined by immunoblotting. (B and C) PLC $\beta$ and PKC were required for G1-induced TAZ activation. Serum-starved ZR-75-30 cells were pretreated with PLC $\beta$ inhibitors (U73122 or ET-18-OCH3) or PKC inhibitors (enzastaurin or chelerythrin chloride) for 4 hours and then stimulated with $100 \mathrm{nM}$ G1 for 2 hours. The lysates were subjected to immunoblot analysis with the indicated antibodies. (D) Inactivation of Rho prevented TAZ dephosphorylation and accumulation following G1 stimulation. Serumstarved ZR-75-30 cells were pretreated with C3 for 4 hours (left panel) or transfected with dominant-negative RhoN19 or Rho GDI (right panel) and then stimulated with $100 \mathrm{nM}$ G1 for 2 hours. Cell lysates were subjected to immunoblotting with the indicated antibodies. (E) ROCK was required for G1-induced TAZ activation. After serum starvation, ZR-75-30 cells were pretreated with the ROCK inhibitor GSK429286 or Y27632 for 4 hours, followed by treatment with $100 \mathrm{nM}$ G1 for 2 hours. Immunoblotting was performed. (F) Disruption of the actin cytoskeleton blocked G1-induced TAZ activation. Serum-starved ZR-75-30 cells were pretreated with Lat B or Cyto D for 15 minutes and then stimulated with 100 nM G1 for 2 hours. Immunoblotting was performed. Data are representative of at least 3 independent experiments. 
A

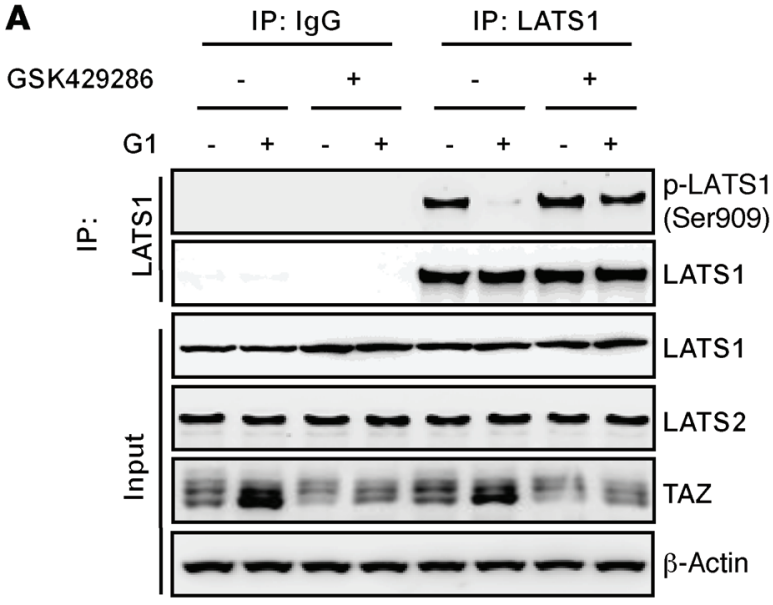

C GSK429286

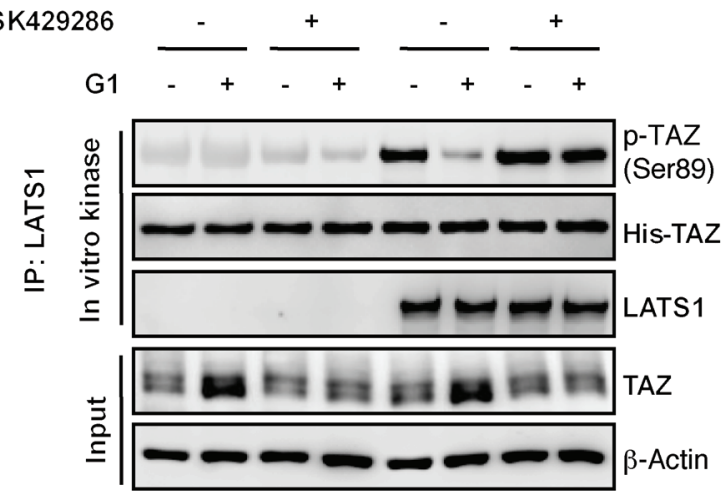

B

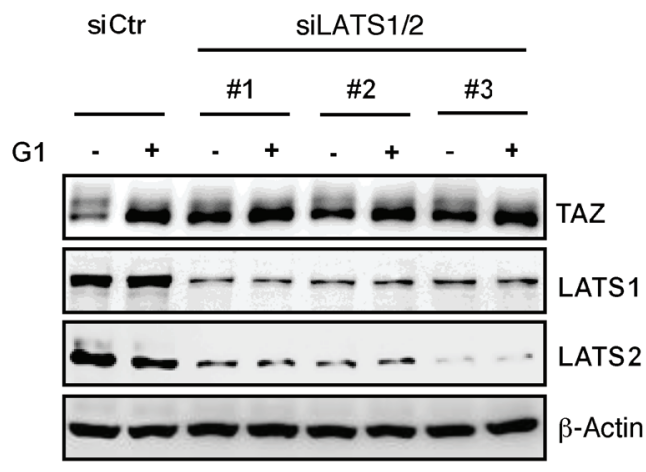

D
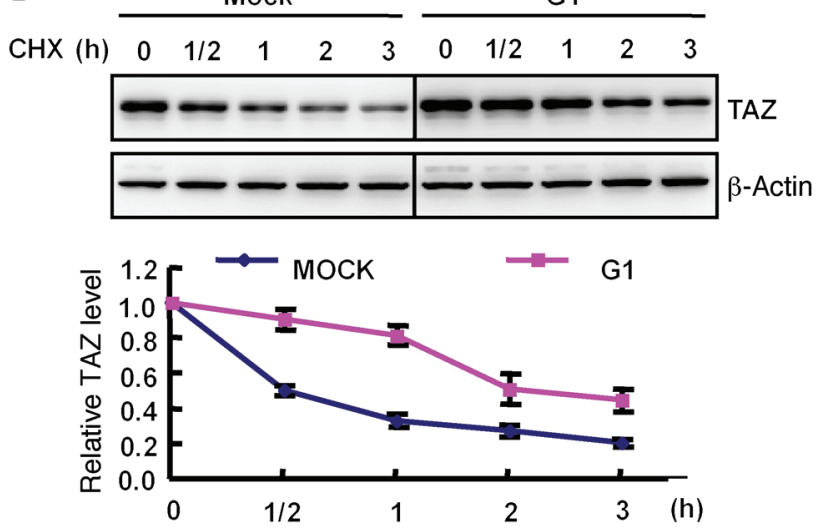

Figure 4. GPER activates TAZ via LATS inhibition. (A) G1 inhibited the phosphorylation of LATS. Serum-starved ZR-75-30 cells were pretreated with 200 nM of the ROCK inhibitor GSK429286 for 4 hours, followed by treatment with 100 nM G1 for 2 hours. For detection of LATS1 phosphorylation, immunoprecipitated LATS1 was used for immunoblotting with p-LATS antibody. (B) LATS was required for G1-induced TAZ accumulation. LATS1/2 were knocked down by 3 independent siRNAs in ZR-75-30 cells. These cells were stimulated with $100 \mathrm{nM}$ G1 as indicated. Protein levels of TAZ and the knockdown efficiency of LATS1/2 were assessed by immunoblotting. (C) LATS1 kinase activity was inhibited by G1 in a ROCK-dependent manner. ZR-75-30 cells were pretreated with the ROCK inhibitor GSK 429286 or control, followed by a 2-hour treatment with 100 nM G1 as indicated. Immunoprecipitated LATS1 was subjected to an in vitro kinase assay using His-TAZ as a substrate. TAZ phosphorylation was detected by p-TAZ (Ser89) antibody. (D) G1 treatment stabilized TAZ protein. Serum-starved ZR-75-30 cells were pretreated with mock or $100 \mathrm{nM} \mathrm{G1}$ for 2 hours and then treated with CHX (20 $\mu \mathrm{g} / \mathrm{ml})$ for the indicated durations. The amounts of TAZ were analyzed by immunoblotting and quantified by densitometry and normalized to $\beta$-actin. Data are represented as the mean \pm SD; $n=3$. Blots shown are representative of at least 3 independent experiments.

overexpressed dominant-negative Rho-N19 and GDI (Rho-GDP dissociation inhibitor), which inhibit endogenous Rho signaling, in ZR-75-30 cells. We found that both Rho-N19 and GDI could block G1-induced TAZ accumulation and CTGF induction (Figure 3D). We consistently found that pretreatment with ROCK inhibitors (GSK429286 or Y27632) also strongly suppressed G1-induced TAZ accumulation, dephosphorylation, nuclear localization, and CTGF induction (Figure 3E and Supplemental Figure 3E). Collectively, these observations suggest a model in which Rho and ROCK serve as important mediators downstream of GPER and are required for GPER to activate TAZ.

The Rho/ROCK pathway potently modulates cellular actin dynamics, particularly stress fiber formation, in response to various GPCR agonists such as LPA or thrombin $(42,43)$. We therefore examined whether cytoskeletal reorganization contributes to TAZ activation in response to G1 stimulation. Treatment with latrunculin B (Lat B) or cytochalasin D (Cyto D), two
F-actin-destabilizing agents, blocked G-induced TAZ dephosphorylation, nuclear localization, and CTGF induction in ZK-7530 cells (Figure 3F and Supplemental Figure 3F). Moreover, G1-induced actin stress fiber and TAZ activation were blocked by C3, GSK429286, or Lat B (Supplemental Figure 3, D-F). Taken together, these data support a model wherein GPER acts through Rho/ROCK and actin organization to modulate the Hippo pathway, leading to eventual TAZ activation.

G1 inhibits LATS, but not MST, in the Hippo pathway. MST1/2 and LATS1/2 are core components of the Hippo pathway and form a kinase cascade to regulate YAP/TAZ phosphorylation and function $(17,44)$. Previous studies have demonstrated that the phosphorylation of Thr183/Thr180 in MST1/2 is critical for their kinase activities (45). We found that neither the phosphorylation levels of MST1 (Thr183)/MST2 (Thr180) nor the protein levels of MST1/2 were changed following G1 treatment (Supplemental Figure 4A). Furthermore, MST1/2 knockdown did not 
A

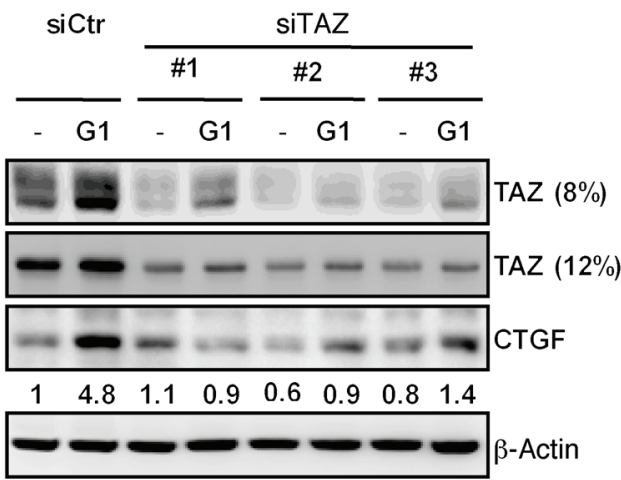

C

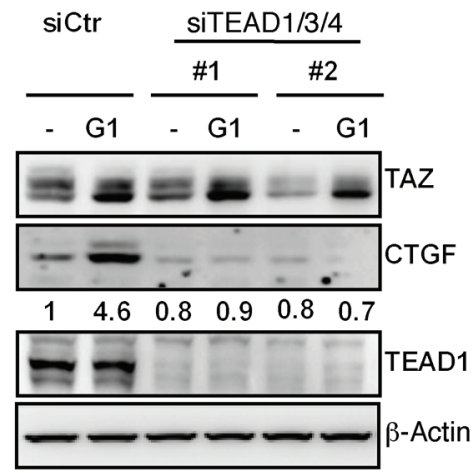

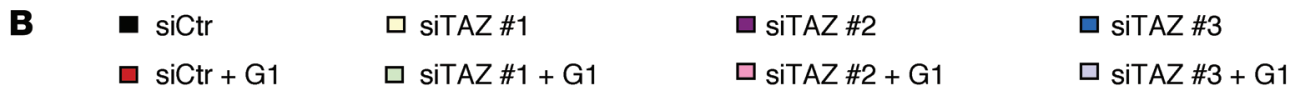

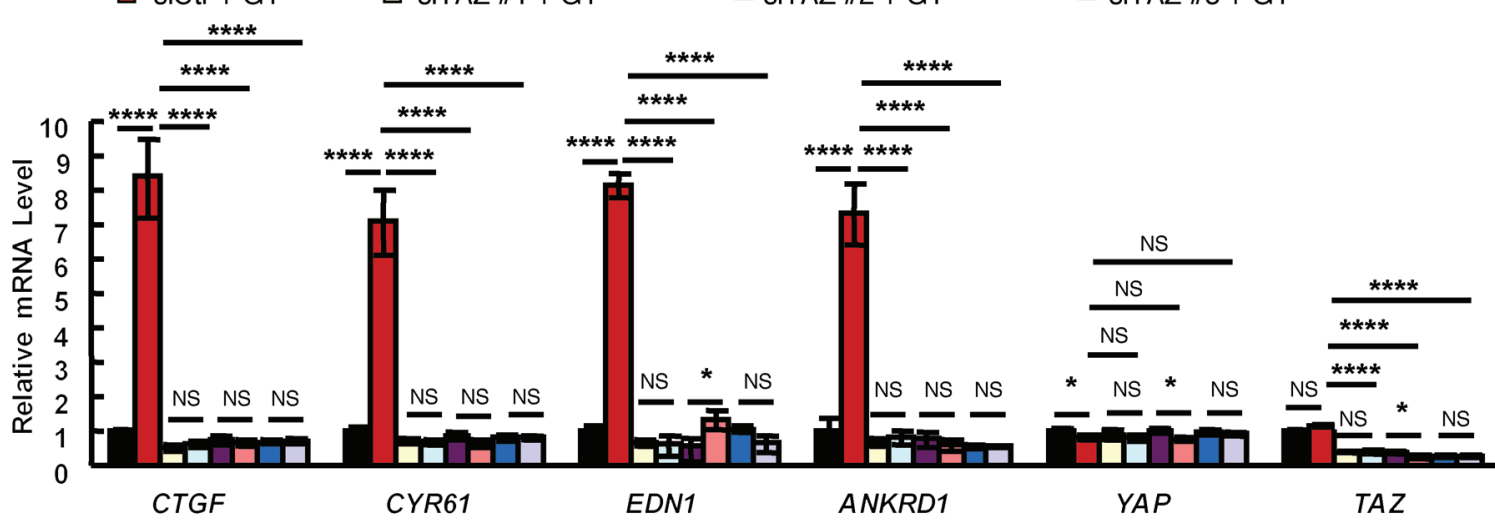

D
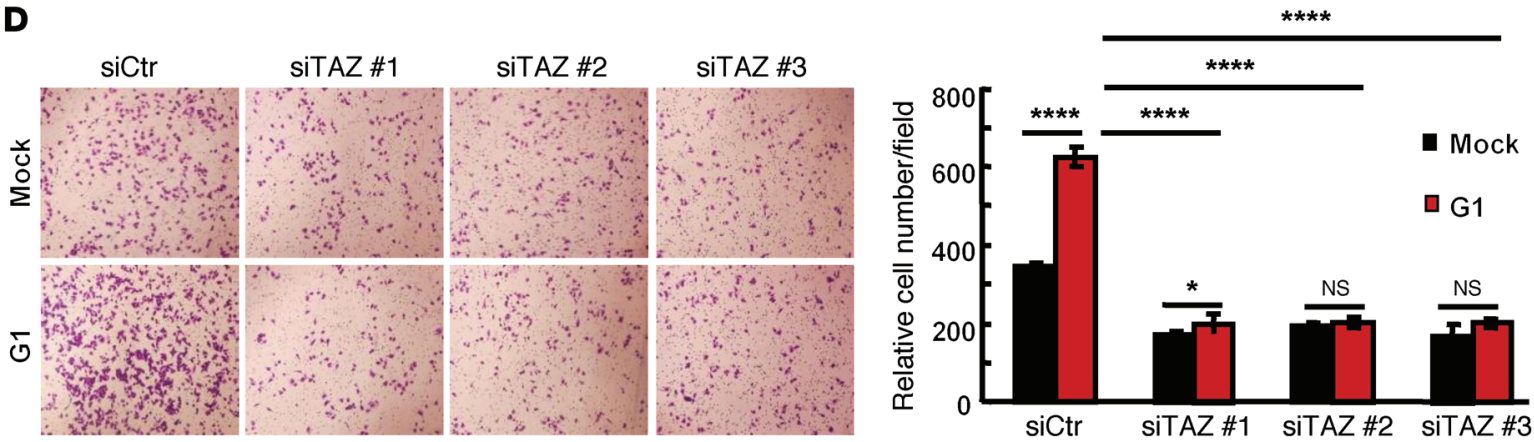

Figure 5. YAP/TAZ and TEAD mediate the physiological functions of GPER activation in target gene induction and cell migration. (A and B) TAZ was required for GPER to induce target gene expression. ZR-75-30 cells were transiently transfected with control or TAZ siRNAs. Serum-starved cells were treated with $100 \mathrm{nM}$ G1 for 2 hours as indicated. Protein levels of CTCF and the knockdown efficiency of TAZ were determined by immunoblotting. In A, $8 \%$ and $12 \%$ refer to the acrylamide concentration in SDS-PACE. mRNA levels of the indicated target genes were measured by quantitative PCR. Data represent the mean $\pm S D ; n=3$. (C) TEADs were indispensable for GPER to induce target gene expression. The experiments were similar to those depicted in A, except TEAD1/3/4 were knocked down by siRNAs. (D) Knockdown of TAZ impaired G1-induced cell migration. ZR-75-30 cells were transiently transfected with the indicated siRNAs and stimulated with $\mathrm{G} 1$ or vehicle for 6 hours after serum starvation. Cell migration was determined by Transwell cell migration assay (50,000 cells/well). Cells were stained with crystal violet (left panels) and quantified (right panel). Data represent the mean \pm SD; $n=5$. Data were analyzed by 1-way ANOVA with LSD correction. ${ }^{*} P<0.05 ;{ }^{* * *} P<0.0001$. Blots shown are representative of at least 3 independent experiments.

affect G1-induced TAZ accumulation (Supplemental Figure 4B). These results suggest that MST is not required and might not be involved in G1-induced TAZ activation.

LATS1/2 are phosphorylated in the activation loop (Ser909/ Ser872 for LATS1/LATS2), which correlates with LATS1/2 activity (44). Upon G1 treatment, we observed a robust decrease in the phosphorylation levels of LATS1 (Ser909) (Figure 4A). Furthermore, pretreatment with the ROCK inhibitor GSK429286 largely blocked the inhibitory effect of G1 on LATS1 (Ser909) phosphorylation (Figure 4A). These data are consistent with the observed function of ROCK in G1-induced TAZ activation. We also found that G1-induced TAZ protein accumulation depends on LATS, as shown by the RNA interference experiments (Figure $4 \mathrm{~B})$. Next, we directly measured the kinase activity of LATS1 immunoprecipitated from control or G1-treated cells. As shown in Figure 4C and Supplemental Figure 4C, LATS1 
A

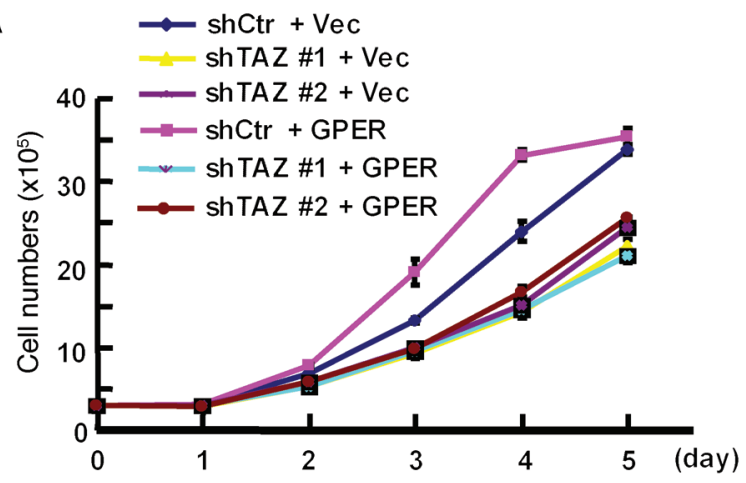

C
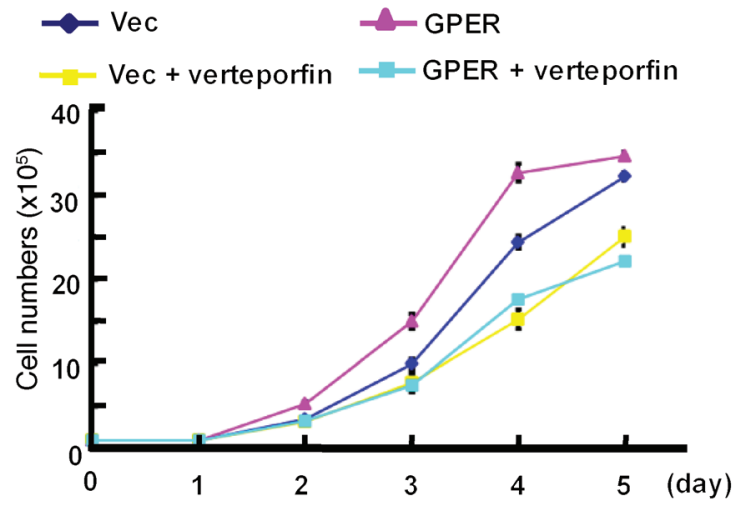

D

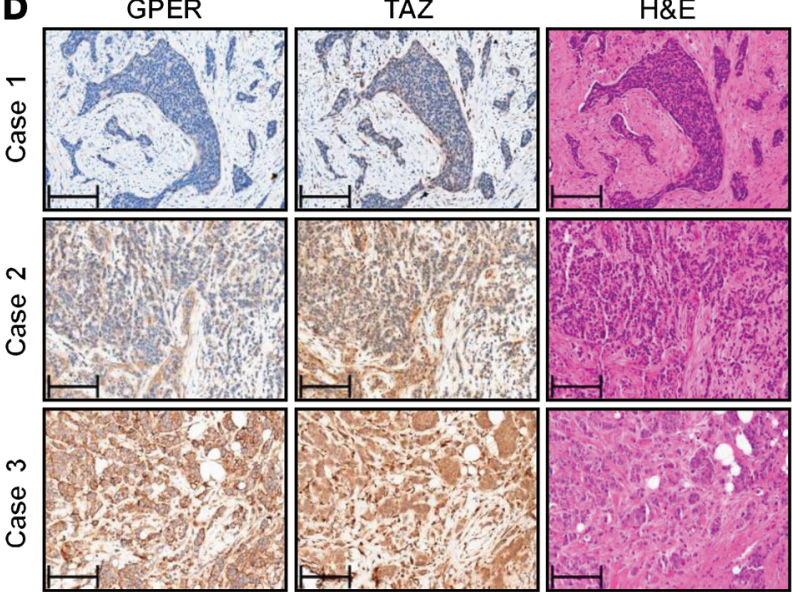

B
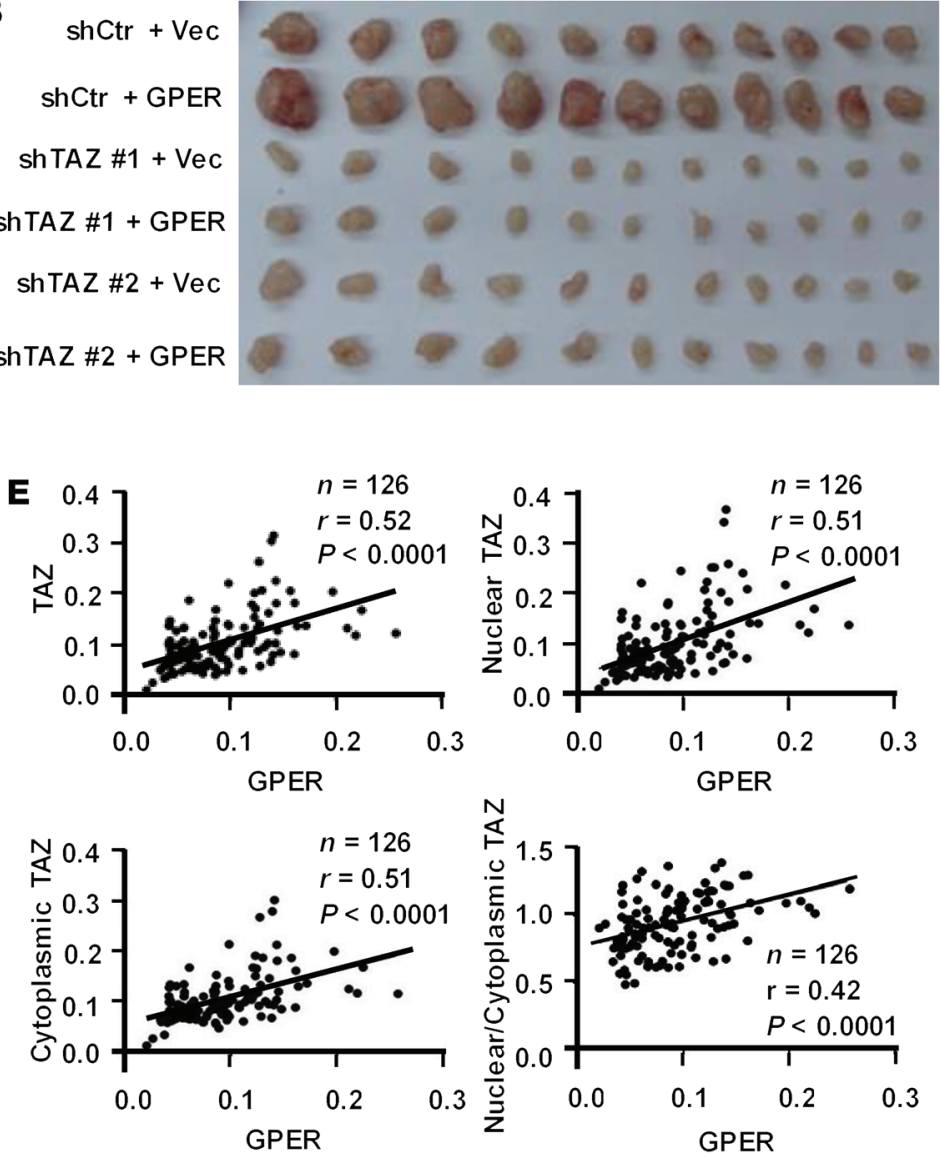

$\mathbf{F}$

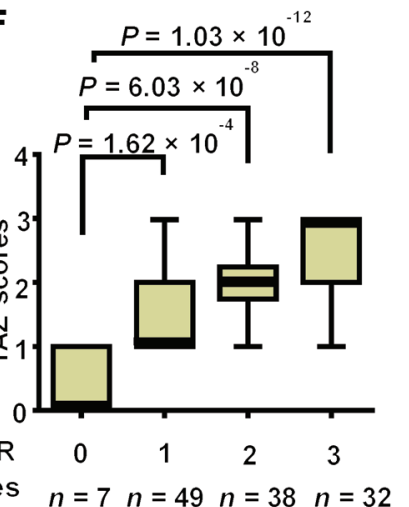

scores $n=7 \quad n=49 \quad n=38 \quad n=32$

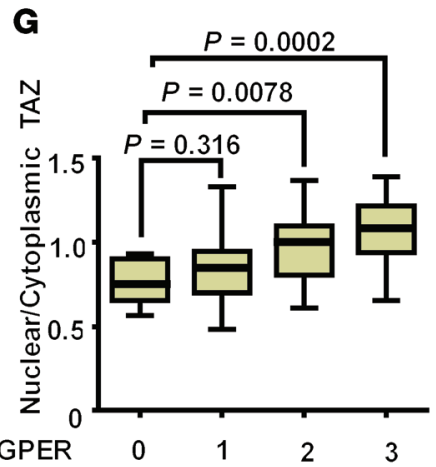

Figure 6. TAZ mediates the tumorigenic effect of GPER, and its expression correlates with GPER in IDCs of the breast. (A) GPER promoted cell proliferation through TAZ. TAZ was knocked down in control or GPER-overexpressing ZR-75-30 cells. Cell proliferation was determined. Error bars represent cell numbers \pm SD for triplicate experiments. (B) TAZ was required for GPER to promote tumor growth in a xenograft mouse model. Xenografting was performed in female nude mice using the cells described in $\mathbf{A}$. Four weeks after injection, tumors from mice were extracted and photographed. (C) Verteporfin blocked GPER-driven cell proliferation. ZR-75-30 cells were treated with $10 \mu \mathrm{M}$ verteporfin, and cell proliferation was measured; $n=3$. Error bars represent cell numbers \pm SD. ( $D$ and $\mathbf{E}$ ) Positive correlation between GPER and TAZ expression in breast IDCs. Images show IHC staining of GPER and TAZ in 2 serial sections of 3 representative tumors. Scale bars: $50 \mu \mathrm{m}$. Total TAZ, cytoplasmic TAZ, nuclear TAZ, nuclear/cytoplasmic TAZ ratio, and GPER expression were analyzed by SPSS linear regression, and the standardized coefficient value is shown. (F and $\mathbf{G})$ The 126 subjects were divided into 4 groups on the basis of their tumor GPER expression scores. Data were analyzed by 1-way ANOVA with LSD correction. Horizontal lines represent the median, and the top and bottom of the boxes represent the 75 th and 25 th percentiles, respectively. Vec, vector.

kinase activity, indicated by the in vitro phosphorylation of purified His-TAZ and GST-YAP, was strongly inhibited upon G1 treatment. Consistent with the LATS1 phosphorylation data in Figure 4A, inhibition of ROCK by GSK 429286 also blocked the effect of G1 on LATS1 kinase inactivation (Figure 4C). Ser89 and Ser311 are 2 sites that are directly phosphorylated by LATS in TAZ $(21,24)$. Consistent with LATS inhibition, G1 treatment reduced the phosphorylation of Ser89 and Ser311 in transfected Flag-TAZ (Supplemental Figure 4D). Collectively, the above results suggest a model in which GPER inhibits LATS in a man- 
ner independent of MST to induce TAZ dephosphorylation and accumulation, and ROCK has an essential role in mediating the GPER signaling to inhibit LATS and activate TAZ.

The accumulation of TAZ by GPER activation could be transcriptional or posttranscriptional. We have previously shown that TAZ is an unstable protein and is degraded via the $26 \mathrm{~S}$ proteasome once it is phosphorylated by LATS (24). As shown in Supplemental Figure 4E, G1 did not significantly increase TAZ mRNA levels. On the other hand, the half-life of TAZ protein was prolonged upon G1 treatment in ZR-75-30 cells (Figure 4D). Consistently, the TAZ ${ }^{4 S A}$ mutant, which has mutations of all 4 LATS phosphorylation sites and cannot be regulated by LATS, was no longer accumulated, whereas the WT TAZ was accumulated in response to G1 stimulation (Supplemental Figure 4F). Combined with the data in Figure $2 \mathrm{~B}$, we propose that activation of GPER leads to the dephosphorylation and stabilization of TAZ.

Activation of TAZ is required for GPER to stimulate target gene expression and cell migration. As shown in Figure $2 \mathrm{G}$ and Supplemental Figure 2D, expression of the well-characterized YAP/TAZ target genes CTGF, CYR61, EDN1, and ANKRD1 were upregulated by G1 stimulation. To further dissect the relative contribution of YAP and TAZ in mediating GPER-activated gene transcription, we silenced YAP or TAZ individually. As shown in Figure 5, A and B, $T A Z$ knockdown was sufficient to block transcription of the target genes in response to GPER stimulation in ZR-75-30 cells, and YAP knockdown had a minor effect on G1-induced CTGF induction and gene transcription (Supplemental Figure 5, A and B). Collectively, these results suggest that TAZ plays a more critical role in G1induced gene induction in ZR-75-30 cells. These results are consistent with the fact that TAZ is abundantly expressed in ZR-75-30 cells (Figure 2G). G1-induced gene expression also required TEAD, as shown in the TEAD1/3/4-knockdown experiments (Figure $5 C$ ). These observations are consistent with a role of TAZ and TEAD in GPER-induced gene expression, at least for the expression of CTGF, CYR61, EDN1, and ANKRD1. However, we cannot exclude the possibility that TEAD plays an obligatory role, albeit one that functions parallel to GPER signaling.

Estrogen is known to stimulate breast cancer cell migration and metastasis through various mechanisms $(9,46)$. YAP/TAZ are also known to promote cell migration $(27,29)$. We therefore examined whether the function of GPER in promoting ZR-75-30 cell migration depends on TAZ. G1 indeed promoted ZR-75-30 cell migration, and this effect was blocked by $T A Z$ knockdown (Figure 5D). In line with the above results, TEAD1/3/4 knockdown impaired G1-induced cell migration (data not shown), supporting a functional role of TAZ and TEAD in GPER signaling to induce breast cancer cell migration.

$T A Z$ mediates the tumorigenic effect of GPER in vivo and is upregulated along with elevated GPER in IDC. We investigated the function of TAZ in GPER-induced tumor cell proliferation. ZR-75-30 cells, which have endogenous GPER with stable overexpression of GPER, were established (Supplemental Figure 6A). Consistent with previous reports $(9,11)$, ectopic expression of GPER enhanced ZR-75-30 cell proliferation (Figure 6A). The GPER-induced cell proliferation was blocked by $T A Z$ knockdown. A xenograft experiment was performed to determine the effect of GPER and TAZ on tumor formation of ZR-75-30 cells. Ectopic expression of GPER promoted tumor growth, and $T A Z$ knockdown abolished this effect of GPER (Figure 6B and Supplemental Figure 6B). Complementary knockdown of either GPER or TAZ decreased ZR-75-30 cell proliferation. Importantly, there was no synergistic effect when GPER and TAZ were knocked down simultaneously (Supplemental Figure 6, C and D). These results are consistent with a model in which TAZ acts downstream of GPER and plays an important role in the tumor-promoting activity of GPER. To further support this, we treated the cells with verteporfin, which disrupts the interaction between YAP/TAZ and TEADs and found that verteporfin inhibited the proliferation-promoting effect of GPER (Figure 6C).

Previous studies have reported that TAZ is overexpressed in $20 \%$ of breast cancers, especially in IDC (29). The expression levels and activity of TAZ are frequently upregulated in high-grade metastatic breast cancer (30). Our study also predicts that TAZ protein would be elevated in tumors with high levels of GPER. We therefore examined and compared the expression of TAZ and GPER in IDC specimens. The specificity of the antibody against TAZ was verified by immunocompetition (Supplemental Figure 6E). As shown in Figure 6, D and E, the total protein levels of TAZ and GPER were positively correlated in IDC specimens. We further analyzed the levels of cytoplasmic and nuclear TAZ separately. As shown in Figure 6E, both cytoplasmic and nuclear TAZ were positively correlated with GPER in 126 cases of human IDC of the breast. In addition, the ratio of nuclear/cytoplasmic TAZ was also positively correlated with GPER expression (Figure 6E). This observation is consistent with our data showing that stimulation of GPER increases TAZ protein levels (by inhibiting degradation) and promotes TAZ nuclear localization (by promoting dephosphorylation) in breast cancer cells.

To better understand this observation, the IDC specimens were divided into 4 groups according to GPER levels, and the expression scores of TAZ were compared among the 4 groups. As shown in Figure 6, F and G, the median score of TAZ or the ratio of nuclear/cytoplasmic TAZ in subjects with high GPER expression was higher than that in subjects with low GPER expression. Similarly, the percentage of the TAZ ${ }^{\text {hi }}$ population was much larger in the GPER ${ }^{\text {hi }}$ group than in the GPER ${ }^{\text {lo }}$ group $\left(P=2.43 \times 10^{-9}\right)$ (Supplemental Figure 6F). These data are consistent with the model that GPER increases TAZ expression in breast cancers and provide clinical evidence supporting a functional relationship between GPER and TAZ.

\section{Discussion}

Estrogen has broad physiological and pathological functions, most notably in the development and maintenance of the female reproductive system and breast cancer (47). Traditionally, estrogen is known to act through 2 classical hormone-activated transcription factors, $\operatorname{ER} \alpha$ and $\operatorname{ER} \beta(1,2)$, which mediate the genomic effects (transcription regulation) of estrogen. Moreover, the seven-transmembrane receptor GPER also mediates estrogen function by modulating both the rapid nongenomic signaling events and the genomic transcriptional effects of estrogen (4, 7-9). In this study, we show that estrogen and other synthetic ligands activate YAP/TAZ, 2 transcription coactivators and oncoproteins downstream of the Hippo pathway, through GPER but not ERs. Our data indicate that Gaq/11, PLC $\beta$, PKC, Rho GTPases, and ROCK act downstream of GPER to inhibit LATS kinase, which is responsible for the phospho- 
rylation and inhibition of YAP/TAZ. The Hippo/YAP/TAZ pathway, therefore, likely plays an important role in mediating the physiological function of GPER in gene expression and cell proliferation.

Transcriptional regulation of estrogen has long been attributed to the classic nuclear estrogen-responsive receptors $\mathrm{ER} \alpha$ and $\operatorname{ER} \beta(1,2)$, but the monopolized model has been challenged since GPER was documented to be involved in the genomic effects of estrogen, albeit indirectly $(8,9)$. It has been reported that FOS and CTGF are 2 target genes of GPER activation through the EGFR/ ERK pathway, and this process does not require ERs (9). Our study suggests a critical role of TAZ in mediating gene transcription upon GPER stimulation. For example, knockdown of either TAZ or TEAD1/3/4 blocks G1-induced CTGF expression (Figure 5, A and $\mathrm{C}$ ). Moreover, the expression of several other genes that are regulated by GPER, such as CYR61, EDN1, and ANKRD1, is also dependent on TAZ (Figure 5B). Thus, the Hippo/YAP/TAZ pathway plays a role in mediating the genomic effects of GPER. We propose that although the majority of estrogen-inducible genes may depend on ERs, some inducible genes may be codependent on ERs and GPER, while others may depend entirely on YAP/TAZ activation in response to estrogen. Future work is needed to clarify the relative contribution and possible collaboration of ERs and GPER in estrogen-induced gene expression.

Estrogen modulates breast epithelial cell proliferation and also plays a prominent role in the development of breast cancer. As such, inhibition of estrogen signaling (either by blocking estrogen production or inhibiting ER function) is a common clinical approach to treat ER-positive breast cancer. However, the relationship between GPER and breast cancer is less clear (12-15). Here, we found that GPER expression was elevated in IDC specimens when compared with the adjacent normal breast epithelial cells. Given the known oncogenic function of YAP/TAZ, we propose that activation of GPER also contributes to breast cancer, at least in part by activating YAP/TAZ, which can stimulate cell proliferation and inhibit apoptosis. Consistently, we have noticed that TAZ protein levels correlate with GPER expression in IDC specimens, supporting the model of TAZ activation by GPER in clinical samples. Although elevated, the expression of GPER did not increase further along with breast cancer progression to advanced stages. Our observation suggests that high levels of GPER contribute to IDC initiation by activating YAP/TAZ, and thus GPER may be a potential biomarker for IDC of the breast.

Tamoxifen is a widely used therapeutic drug for hormonedependent breast cancer. However, 25\% of ER-positive patients do not respond to tamoxifen (48). Notably, tamoxifen acts not only as an antagonist of ERs, but also as an agonist of GPER. The same is true for another ER antagonist drug, fulvestrant, which inhibits ERs but stimulates GPER $(7,9,35)$. Therefore, tamoxifen or fulvestrant may have dual effects on breast cancer cells: inhibition by blocking ER and stimulation by activating GPER. This may be a possible mechanism for tamoxifen resistance in some of the ER-positive breast cancers that express high levels of GPER. Consistently, overexpression of GPER in MCF7 breast cancer cells indeed increases tamoxifen resistance (49). Furthermore, there are clinical data showing that GPER is involved in tamoxifen resistance in breast cancer (12). Our observations may have an important implication for breast cancer treatment. ER-posi- tive breast cancers should be further stratified into GPER ${ }^{\text {hi }}$ and GPER $^{\text {lo }}$ subclasses. Tamoxifen will be a good choice for patients who are $\mathrm{ER}^{+} \mathrm{GPER}^{\text {lo }}$; whereas, for $\mathrm{ER}^{+} \mathrm{GPER}{ }^{\text {hi }}$ patients, the effects of tamoxifen could be complicated. The use of drugs that simultaneously block GPER and ER may be a better strategy to treat this subclass of breast cancers. Furthermore, GPER expression strongly correlates with TAZ in IDC samples, indicating that TAZ is highly expressed in GPER ${ }^{\text {hi }}$ subjects (Figure 6, D-G, and Supplemental Figure $6 \mathrm{~F}$ ) and might contribute to tamoxifen resistance. Future studies are needed to clarify the role of GPER and YAP/ TAZ in breast cancer development and tamoxifen resistance.

\section{Methods}

Cohort. In the IDC cohort, a total of 126 formalin-fixed, paraffin-embedded tissue samples were obtained from the tissue bank of Shanghai Medical College of Fudan University. The clinical pathological parameters including IHC staining for ER, PR, and HER2, tumor size, and axillary lymph node metastasis status were included in the bank. Histologic types and clinical stages were also defined according to World Health Organization (WHO) classification criteria.

IHC and quantification. IHC staining was performed as previously described, with some modification (50). Rabbit antibodies against GPER or TAZ used in IHC were diluted at 1:100. To quantify the expression of GPER and TAZ, 5 random views were selected and imaged at $\times 200$ magnification through a liquid crystal filter of the CSI-Nuance Multispectral Imaging System (Cambridge Research and Instrumentation Inc., PerkinElmer) at a wavelength of 420 to $720 \mathrm{~nm}$ in $15-\mathrm{nm}$ increments. inForm software, version 1.4.0 (PerkinElmer), was used for further quantification of DAB intensity of the image cubes. The DAB signal of the cytoplasmic and nuclear TAZ or the membranic GPER was used in this study. The mean value of the signal intensity of each case was categorized into the corresponding groups by the following scores: 0 ( $<0.04$, negative staining); 1 (0.04-0.08, weak staining); 2 (0.08-0.12, moderate staining); and 3 (>0.12, strong staining). Further analysis was based on the IHC scores of GPER and/or TAZ.

Cell migration assay. Cell migration was assayed using BD Falcon Cell culture chambers with $8.0-\mu \mathrm{m}$ pores in 24 -well plates. Serumstarved ZR-75-30 cells were stimulated with G1 or vehicle for 6 hours and suspended in serum-free DMEM. Cells $\left(5 \times 10^{4}\right)$ were seeded in each well, and DMEM supplemented with $10 \%$ FBS was poured into the bottom chamber of the plates. Twenty-four hours after seeding, cells that attached on membranes were fixed in $4 \%$ paraformaldehyde and stained with $0.05 \%$ crystal violet. The migrated cells on the lower surface of the membrane were photographed and quantified.

Cell proliferation assay. For quantitative proliferation assays, $3 \times 10^{5}$ ZR-75-30 stable cells were seeded in 6-well plates in triplicate in regular growth medium. The culture medium was renewed every other day. Cell numbers were counted daily.

Xenograft. The ZR-75-30 stable cells suspended in PBS $\left(5 \times 10^{6}\right)$ were inoculated s.c. into the mammary fat pad of 5- to 6-week-old female BALB/c nude mice, which were purchased from the Shanghai Laboratory Animal Company (SLAC), with 11 nude mice in each group. All mice were sacrificed 4 weeks after injection, and tumor weights were measured.

Statistics. All values are reported as the mean \pm SD. A 1-way ANOVA was used for multiple comparisons. Unless otherwise indicated, comparisons between 2 groups were performed using a 2-tailed 
Student's $t$ test, assuming equal variances between groups. $P$ values of less than 0.05 were considered statistically significant.

Study approval. All experiments involving animals were reviewed and approved by the Animal Welfare Committee of Shanghai Medical College of Fudan University. The procedures related to human subjects were approved by the ethics committee of the Institutes of Biomedical Sciences (IBS) of Fudan University. All patients who participated in the study provided written informed consent.

\section{Acknowledgments}

We thank the members of the Fudan Molecular and Cell Biology laboratory for discussions throughout this study. We also thank the Biomedical Core Facility of Fudan University for technical support. This work was supported by the Ministry of Science and Technology of the People's Republic of China (MOST) (2015CB910401 and 2011CB910601 to Q.Y. Lei; 2012CB910101 to K.L. Guan); the National Natural Science Foundation of China (NSFC) (81430057, 81225016, and 31271454); the Shanghai Key Basic Research Program (12JC1401100); the "100 Talents" Pro- gram of Shanghai Health (XBR2011041); the Scholar of "Dawn" Program of the Shanghai Education Commission; and a Shanghai Outstanding Academic Leader grant (13XD1400600, to Q.Y. Lei). This work was also supported by the 985 Program; the Shanghai Leading Academic Discipline Project (pB110); and by NIH grants (to K.L. Guan and Y. Xiong).

Address correspondence to: Yue Xiong, Department of Biochemistry and Biophysics, Lineberger Comprehensive Cancer Center, University of North Carolina at Chapel Hill, Chapel Hill, North Carolina 27599, USA. Phone: 919.962.2142; E-mail: yxiong@email.unc.edu. Or to: Qun-Ying Lei, Department of Biochemistry and Molecular Biology, School of Basic Medical Sciences, Fudan University Shanghai Medical College, Dong An Road 131, Mingdao Building 701, Shanghai 200032, China. Phone: 8621.54237935; E-mail: qlei@ fudan.edu.cn. Or to: Kun-Liang Guan, Department of Pharmacology and Moores Cancer Center, University of California San Diego, RM5344, 3855 Health Sciences Dr., La Jolla, California 92093-0815, USA. Phone: 858.822.7945; E-mail: kuguan@ucsd.edu.
1. Klinge CM. Estrogen receptor interaction with estrogen response elements. Nucleic Acids Res. 2001;29(14):2905-2919.

2. McDonnell DP, Norris JD. Connections and regulation of the human estrogen receptor. Science. 2002;296(5573):1642-1644.

3. Carmeci C, Thompson DA, Ring HZ, Francke U, Weigel RJ. Identification of a gene (GPR30) with homology to the G-protein-coupled receptor superfamily associated with estrogen receptor expression in breast cancer. Genomics. 1997;45(3):607-617.

4. Revankar CM, Cimino DF, Sklar LA, Arterburn JB, Prossnitz ER. A transmembrane intracellular estrogen receptor mediates rapid cell signaling. Science. 2005;307(5715):1625-1630.

5. Dorsam RT, Gutkind JS. G-protein-coupled receptors and cancer. Nat Rev Cancer. 2007;7(2):79-94.

6. O'Hayre M, et al. The emerging mutational landscape of $G$ proteins and G-protein-coupled receptors in cancer. Nat Rev Cancer. 2013;13(6):412-424.

7. Filardo EJ, Quinn JA, Bland KI, Frackelton AR, Frackelton AR Jr. Estrogen-induced activation of Erk-1 and Erk-2 requires the G protein-coupled receptor homolog, GPR30, and occurs via trans-activation of the epidermal growth factor receptor through release of HB-EGF. Mol Endocrinol. 2000;14(10):1649-1660.

8. Maggiolini M, et al. The $G$ protein-coupled receptor GPR30 mediates c-fos up-regulation by $17 \beta$-estradiol and phytoestrogens in breast cancer cells. J Biol Chem. 2004;279(26):27008-27016.

9. Pandey DP, Lappano R, Albanito L, Madeo A, Maggiolini M, Picard D. Estrogenic GPR30 signalling induces proliferation and migration of breast cancer cells through CTGF. EMBO J. 2009;28(5):523-532.

10. Albanito L, et al. G protein-coupled receptor 30 (GPR30) mediates gene expression changes and growth response to 17beta-estradiol and selective GPR30 ligand G-1 in ovarian cancer cells. Cancer Res. 2007;67(4):1859-1866.
11. Vivacqua A, et al. The G protein-coupled receptor GPR30 mediates the proliferative effects induced by $17 \beta$-estradiol and hydroxytamoxifen in endometrial cancer cells. Mol Endocrinol. 2006;20(3):631-646.

12. Ignatov A, et al. G-protein-coupled estrogen receptor GPR30 and tamoxifen resistance in breast cancer. Breast Cancer Res Treat. 2011;128(2):457-466.

13. Filardo EJ, et al. Distribution of GPR30, a seven membrane-spanning estrogen receptor, in primary breast cancer and its association with clinicopathologic determinants of tumor progression. Clin Cancer Res. 2006;12(21):6359-6366.

14. Kuo WH, et al. The interactions between GPR30 and the major biomarkers in infiltrating ductal carcinoma of the breast in an Asian population. Taiwan J Obstet Gynecol. 2007;46(2):135-145.

15. Poola I, Abraham J, Liu A, Marshalleck JJ, Dewitty RL. The cell surface estrogen receptor, G Protein-coupled Receptor 30 (GPR30), is markedly down regulated during breast tumorigenesis. Breast Cancer (Auckl). 2008;1:65-78.

16. Pan D. Hippo signaling in organ size control. Genes Dev. 2007;21(8):886-897.

17. Zhao B, Li L, Lei Q, Guan KL. The Hippo-YAP pathway in organ size control and tumorigenesis: an updated version. Genes Dev. 2010;24(9):862-874.

18. Harvey KF, Zhang X, Thomas DM. The Hippo pathway and human cancer. Nat Rev Cancer. 2013;13(4):246-257.

19. Zhao B, et al. Inactivation of YAP oncoprotein by the Hippo pathway is involved in cell contact inhibition and tissue growth control. Genes Dev. 2007;21(21):2747-2761.

20. Dong J, et al. Elucidation of a universal sizecontrol mechanism in Drosophila and mammals. Cell. 2007;130(6):1120-1133.

21. Lei QY, et al. TAZ promotes cell proliferation and epithelial-mesenchymal transition and is inhibited by the hippo pathway. Mol Cell Biol. 2008;28(7):2426-2436.

22. Hao Y, Chun A, Cheung K, Rashidi B, Yang $\mathrm{X}$. Tumor suppressor LATS1 is a negative regulator of oncogene YAP. J Biol Chem. 2008;283(9):5496-5509.

23. Zhao B, Li L, Tumaneng K, Wang CY, Guan KL. A coordinated phosphorylation by Lats and CK1 regulates YAP stability through SCF(beta-TRCP). Genes Dev. 2010;24(1):72-85.

24. Liu CY, et al. The hippo tumor pathway promotes TAZ degradation by phosphorylating a phosphodegron and recruiting the SCF $\{$ beta\}-TrCP E3 ligase. J Biol Chem. 2010;285(48):37159-37169.

25. Huang W, et al. The N-terminal phosphodegron targets TAZ/WWTR1 protein for SCF $\beta$-TrCP-dependent degradation in response to phosphatidylinositol 3-kinase inhibition. J Biol Chem. 2012;287(31):26245-26253.

26. Zhao B, et al. TEAD mediates YAP-dependent gene induction and growth control. Genes Dev. 2008;22(14):1962-1971.

27. Zhang $\mathrm{H}$, et al. TEAD transcription factors mediate the function of TAZ in cell growth and epithelial-mesenchymal transition. J Biol Chem. 2009;284(20):13355-13362.

28. Murakami H, et al. LATS2 is a tumor suppressor gene of malignant mesothelioma. Cancer Res. 2011;71(3):873-883.

29. Chan SW, et al. A role for TAZ in migration, invasion, and tumorigenesis of breast cancer cells. Cancer Res. 2008;68(8):2592-2598.

30. Cordenonsi M, et al. The Hippo transducer TAZ confers cancer stem cell-related traits on breast cancer cells. Cell. 2011;147(4):759-772.

31. Yu FX, et al. Regulation of the Hippo-YAP pathway by G-protein-coupled receptor signaling. Cell. 2012;150(4):780-791.

32. Miller E, et al. Identification of serum-derived sphingosine-1-phosphate as a small molecule regulator of YAP. Chem Biol. 2012;19(8):955-962.

33. Riggs BL, Hartmann LC. Selective estrogen-receptor modulators - mechanisms of action and application to clinical practice. $N$ Engl JMed. 2003;348(7):618-629.

34. Osborne CK. Tamoxifen in the treatment of breast cancer. NEngl JMed.1998;339(22):1609-1618. 
35. Thomas P, Pang Y, Filardo EJ, Dong J. Identity of an estrogen membrane receptor coupled to a $G$ protein in human breast cancer cells. Endocrinology. 2005;146(2):624-632.

36. Bologa CG, et al. Virtual and biomolecular screening converge on a selective agonist for GPR30. Nat Chem Biol. 2006;2(4):207-212.

37. Dennis MK, et al. In vivo effects of a GPR30 antagonist. Nat Chem Biol. 2009;5(6):421-427.

38. Lappano R, Maggiolini M. G protein-coupled receptors: novel targets for drug discovery in cancer. Nat Rev Drug Discov. 2011;10(1):47-60.

39. Kurt AH, Buyukafsar K. Vasoconstriction induced by G1, a G-protein-coupled oestrogen receptor1 (GPER-1) agonist, in the isolated perfused rat kidney. Eur J Pharmacol. 2013;702(1):71-78.

40. Zhao B, Li L, Wang L, Wang CY, Yu J, Guan KL. Cell detachment activates the Hippo pathway via cytoskeleton reorganization to induce anoikis.
Genes Dev. 2012;26(1):54-68.

41. Dupont S, et al. Role of YAP/TAZ in mechanotransduction. Nature. 2011;474(7350):179-183.

42. Sawada K, et al. Lysophosphatidic acid induces focal adhesion assembly through Rho/Rho-associated kinase pathway in human ovarian cancer cells. Gynecol Oncol. 2002;87(3):252-259.

43. Ruiz-Loredo AY, Lopez E, Lopez-Colome AM. Thrombin promotes actin stress fiber formation in RPE through Rho/ROCK-mediated MLC phosphorylation. JCell Physiol. 2011;226(2):414-423.

44. Chan EH, Nousiainen M, Chalamalasetty RB, Schafer A, Nigg EA, Sillje HH. The Ste2Olike kinase Mst2 activates the human large tumor suppressor kinase Lats1. Oncogene. 2005;24(12):2076-2086.

45. Glantschnig H, Rodan GA, Reszka AA. Mapping of MST1 kinase sites of phosphorylation. Activation and autophosphorylation. J Biol Chem.
2002;277(45):42987-42996.

46. Prest SJ, May FE, Westley BR. The estrogen-regulated protein, TFF1, stimulates migration of human breast cancer cells. FASEB J. 2002;16(6):592-594.

47. Deroo BJ, Korach KS. Estrogen receptors and human disease. JClin Invest. 2006;116(3):561-570.

48. Early Breast Cancer Trialists' Collaborative Group (EBCTCG). Effects of chemotherapy hormonal therapy for early breast cancer on recurrence 15-year survival: an overview of the randomised trials. Lancet. 2005;365(9472):1687-1717.

49. Ignatov A, Ignatov T, Roessner A, Costa SD, Kalinski T. Role of GPR30 in the mechanisms of tamoxifen resistance in breast cancer MCF-7 cells. Breast Cancer Res Treat. 2010;123(1):87-96.

50. Lei Q, et al. NKX3.1 stabilizes p53, inhibits AKT activation, blocks prostate cancer initiation caused by PTEN loss. Cancer Cell. 2006;9(5):367-378. 